\title{
Research of Node Energy consumption Based on Dynamic Power Management in Wireless Greenhouse Control System
}

\author{
Yongxian Song \\ The Institute of Electronic Engineering \\ Huaihai Institute of Technology \\ Lianyungang, China \\ e-mail: soyox@126.com
}

\author{
Juanli Ma Xianjin Zhang \\ The Institute of Electronic Engineering \\ Huaihai Institute of Technology \\ Lianyungang, China \\ e-mail: soyox@163.com
}

\begin{abstract}
The resources of wireless sensor nodes are limited, and the power of nodes is supplied with battery whose capacity is very limited, once the energy of battery is run out, the nodes will lose their effect, so there are serious energy constraint in wireless sensor network. In this paper, the specific energy consumption of sensor nodes is studied by experiments, the energy experimental hardware platform is established with the low power consumption SCM MSP430F135 and wireless transceiver chip CC2420, the relationship of energy consumption and communication distance for nodes is discussed, and the energy management strategy of effective wireless greenhouse monitoring system based on dynamic power management is put forward and discussed, the mobile structure is adopted in the convergent nodes. The experiment and simulation results show that the energy consumption of nodes is proportional to the third power of communication distance, the communication distance of sensor nodes can greatly shorten, and energy consumption is saved.
\end{abstract}

Keywords-WSN; Dynamic power management; energy consumption

\section{INTRODUCTION}

Wireless Sensor Network (WSN) integrated sensor technology, embedded computing technology, modern Network, wireless communication technology and distributed information processing technology, etc, all kinds of environment or information of monitoring objects are realtime monitored, perceived and collected through all kinds of integrated micro sensors, after it is processed by the embedded system, and the perceived information will be transferred to the user terminal through the random selforganized wireless communication network by means of multi-hop relay. But the bulk of sensor nodes is small, the capacity is limited, and the power usually is supplied with batteries, so the network has serious energy constraint. Especially in the severe environment, the most sensor nodes are still, it is very hard to replace battery once the nodes are put into use, and can't meet the requirements of its survival time. Therefore, the core issues of hardware design are how to save the battery energy of wireless sensor network nodes, reduce the energy consumption of wireless sensor network when the functions of WSN are affected, and it is also a focus subject at domestic and foreign now[4] . In order to maximize the life cycle of wireless sensor network, the limited energy of nodes must be effectively used when the wireless sensor network circuit, system structure, algorithm and agreement are designed, although the system design has completed , the energy of system operation may be saved by Dynamic Power Management (DPM) technology and Dynamic Voltage scheduling technology (DVS), and the life cycle of sensor system may be prolonged .

In the energy saving mode of DVS, when calculating load is lower, the energy consumption of the microprocessor is saved by reducing working voltage and frequency, DPM can effectively reduce the system power when the performance conditions of system are affected, the internal each function module of sensor nodes is awakened and in energy consumption state when it is only necessary [1-3] . In this paper, the wireless greenhouse control system is taken as application background, the sensor nodes power consumption and state transition conditions are studied, the specific relations of the nodes energy consumption and communication distance are discussed, and the energy management strategy of the wireless greenhouse monitoring system based on dynamic power management is proposed.

\section{DYNAMIC ENERGY MANAGEMENT STRATEGY ANALYSIS}

The nodes usually consist of sensor module, data processing module and communication module in wireless sensor network, and the energy consumption consists of the three parts. The energy consumption of sensor relates with specific application. Calculation of energy consumption include the two parts, that is , energy consumption of processors and memory, and it closely related with the hardware design of sensor nodes and calculation models. Now, the main methods to reduce energy consumption calculation adopt low energy consumption devices and energy perception mode in the node operating systems. The energy consumption of sensor nodes are mainly consumed in wireless communication module, and the energy-saving of network layer protocol is mainly for wireless communication module, so we have to further study relation of nodes energy consumption and communication distance. At present, the relation of wireless communication energy consumption and 
communication distance is $E=k d^{n}$ in the wireless sensor network, and $2<n<4$. The value of $n$ is related with a lot of factors, for example, the deployment of sensor nodes is close to the ground, the more obstacles are, interference is bigger, and the values increase too. Air humidity and antenna quality have much greater influence on

the quality of signal emission. Considering many factors, $n$ is usually taken as 3 . Therefore, with the increase of the communication distance, the energy consumption of nodes will be increased dramatically by way of series. So when the communication connectivity is satisfied, the single jump communication distance should be shortened as soon as possible.

\section{A. Node layer DPM.}

According to dynamic power management strategy of literature [6], the DPM strategies of nodes are as follows: Assume that nodes detect an event, and this event is processed in $t_{1}$ moment, the next event happen in $t_{2}=t_{1}+t_{i}$ moment. So, nodes can keep the depth of sleep as far as possible in idle period, the nodes decided to enter sleep $S_{k}$ in $t_{1}$ moment. The power consumption of sleep $S_{k}$ is $P_{k}$ for each node, $\tau_{0, k}$ and $\tau_{k, 0}$ is time from the working state $S_{0}$ to sleep $S_{k}$ and from sleep $S_{k}$ waken up to the working state $S_{0}$ respectively. For any $i<j$, and $P_{i}>P_{j}, \tau_{0, i}<\tau_{0, j}$ and $\tau_{i, 0}<\tau_{j, 0}$. The work state transition for nodes is shown in Figure.1, the saving energy in state transition may express by formula (1).

$$
\begin{aligned}
& E_{\text {save }, k}=\frac{1}{2}\left(P_{0}-P_{k}\right)\left(t_{i}-\tau_{0, k}+t_{i}-\tau_{k, 0}\right) \\
& =\left(P_{0}-P_{k}\right)\left(t_{i}-\frac{\tau_{0, k}+\tau_{k, 0}}{2}\right)
\end{aligned}
$$

It is shown by formula(1) that the greater power consumption gap ( $\left.P_{0}-P_{k}\right)$ in two state is, the idle time $t_{i}$ is longer, the more shorter state transition time $\tau_{0, k}$ and $\tau_{k, 0}$ are, and the more energy saving of state transition is. This is fully complied with the actual situation. Due to need carry out field information preservation and extraction in the state transition process for nodes, and we will take energy consumption of it as $\Delta E$, supposed that the state transition minimum threshold time of nodes is $T_{t h, k}$, it is as follows.

$$
T_{t h, k}=\frac{1}{2}\left(\tau_{0, k}+\tau_{k, 0}\right)+\frac{\Delta E}{P_{0}-P_{k}}
$$

It is shown by formula (2) that $\tau_{0, k}+\tau_{k, 0}, P_{0}-P_{k}$, and $\Delta E$ only related with equipment itself electrical characteristics, and have nothing to do with the task request of outside world. Therefore, $T_{t h, k}$ of equipment can only be determined.

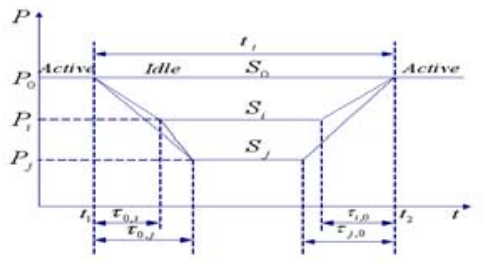

Figure.1 Node work state transition diagram

\section{B. Network layer DPM}

The network layer DPM can reduce activity nodes of wireless sensor network and sensor nodes transmitting power in the premise of guaranteeing the network connectivity, so the energy consumption for whole network system is reduced, in order to prolong working life of the network system. Due to environmental parameter acquisition rate is not high in the greenhouse control system, on the basis of the DPM strategy thoughts, the convergent nodes use mobile work way, the power energy of convergent nodes is unlimited( the energy is supplied with electric power) and has strong communication ability, they move back and forth on top of the greenhouse fixed guide, and communicate with the sensor nodes (or control nodes) in turn, in order to complete the information acquisition and transfer control command task.

Due to the convergent nodes work by means of moving, the communication distance of sensor nodes is greatly shorten, the relationship of energy consumption of wireless communication and communication distance is $E=k d^{n} \quad(2<\mathrm{n}<4)$, so the power consumption of sensor nodes will be decreased by series. When the power consumption of sensor nodes is reduced, the number of convergent nodes is reduced due to adopting the moving work mode. In order to reduce activities sensor nodes quantity, convergent nodes and sensor nodes form a kind of dynamic star network by means of edge to edge. When convergent nodes move to a certain position, a temporary star network is formed, according to the physical location relationship between the sensor nodes and convergent nodes and the moving trend of convergent nodes, the communication channel that is easily out of convergent nodes communication range is arranged first, and the each sensor node GTS communication channel is distributed in the premise of no loss any sensor nodes information. After the information collection is over, and the convergent nodes move to the next position to form a new temporary star network information acquisition. 
The convergent nodes communicate with the monitoring center by the way of multiple hops. The convergent nodes will sent the acquisition information of the greenhouse environment to the monitoring center through multiple hops links. The monitoring center process information data from convergent nodes, and implement the control algorithm, according to the crop growth best conditions and the current greenhouse environment, the control strategy is made, and control commands are sent to converge nodes. The control commands are forwarded to control nodes by convergent nodes, then the control nodes control corresponding actuator and complete control tasks according to the control commands.

\section{EXPERIMENTAL RESEARCH AND ANALYSIS}

\section{A. Design of experimental platform}

In greenhouse wireless sensor network measurement and control system, the convergent node contains processor module, wireless communication module and continuous power supply module. Control nodes and convergent nodes have the same module structure. The structure of transceiver nodes in the monitoring center is similar to that of convergent nodes, but it is supplied with PC of the monitoring center power. Compared with the convergent nodes, the sensor nodes increased the sensor module and the switch that can be set up their own physical location information position, the energy module is supplied with battery. Sensor module is responsible for monitoring area information collection and data conversion, according to the application requirements, the temperature sensor, humidity sensor, light sensor, carbon dioxide concentration sensor and so on can be chosen. The processor module is responsible for the control of the whole sensor nodes operation, storage, processing acquisition data of the node and other nodes. Wireless communication module is responsible for wireless communication between this node and other nodes, exchange control information and data transceiver; Position switch is used to set sensor nodes specific physical location in the greenhouse; the energy module provides the energy for sensor nodes, and is supplied with miniature batteries, the system structure of sensor nodes is shown in Figure. 2.

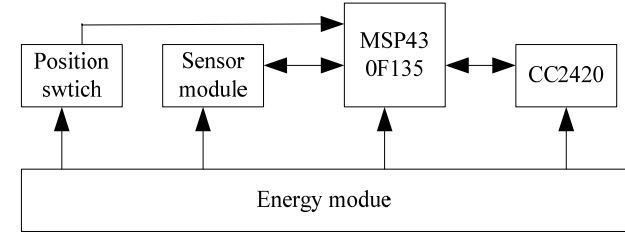

Figure.2. The system structure of sensor nodes

\section{B. Experimental analysis}

Dynamic power management is realized by means of reasonably switching work state of nodes. Therefore, it is very important to the state transfer time consumption and energy consumption of dynamic power management strategy. The timing measurement principle to use Timer _ A of MSP430F135 to state conversion of nodes is shown in Figure.3.
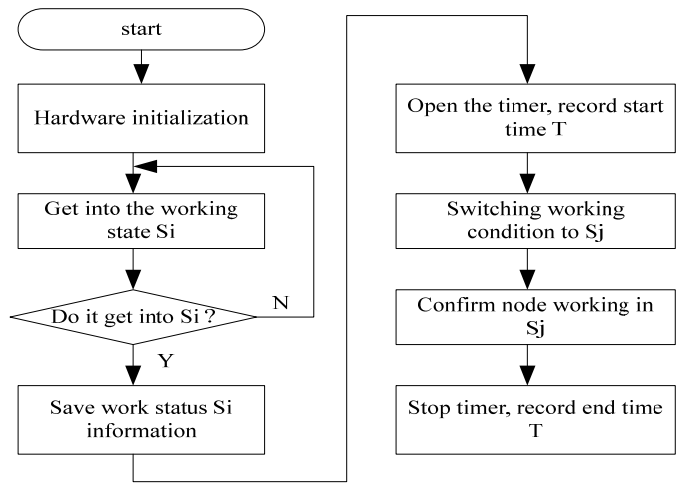

Figure.3. State transition time measuring process

The each state switching time consumption that obtained by experiment is shown in Table 1.

TABLE 1 NODES STATE TRANSITION TIME

\begin{tabular}{|c|c|c|c|c|c|}
\hline$\underbrace{\substack{\text { orignal } \\
\text { state }}}_{\substack{\text { conversion on } \\
\text { state }}}$ & so & s1 & $\mathrm{s} 2$ & s3 & 54 \\
\hline so & oms & $1.476 \mathrm{~ms}$ & $1.540 \mathrm{~ms}$ & $1.470 \mathrm{cms}$ & $1.540 \mathrm{~ms}$ \\
\hline $\mathrm{s} 1$ & $0.128 \mathrm{~ms}$ & $0 \mathrm{~ms}$ & $0.136 \mathrm{~ms}$ & $0.072 \mathrm{~ms}$ & $0.136 \mathrm{~ms}$ \\
\hline $\mathrm{s} 2$ & $1.532 \mathrm{~ms}$ & $1.468 \mathrm{~ms}$ & Oms & $1.404 \mathrm{~ms}$ & $1.468 \mathrm{~ms}$ \\
\hline$s_{3}$ & $0.128 \mathrm{~ms}$ & $0.064 \mathrm{~ms}$ & $0.064 \mathrm{~ms}$ & Oms & $0.064 \mathrm{~ms}$ \\
\hline 54 & a. $192 \mathrm{~ms}$ & a. $128 \mathrm{~ms}$ & $0.128 \mathrm{~ms}$ & $0.064 \mathrm{~ms}$ & $0 \mathrm{~ms}$ \\
\hline
\end{tabular}

Assume that the time ratio of work ( $S_{0}$ and $\left.{ } S_{1}\right)$ and rest

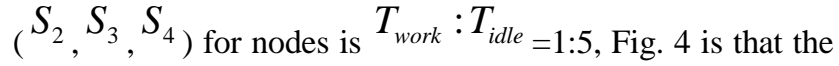
state $S_{1}$ has influence on working life of nodes, ( $S_{2}: S_{3}: S_{4}=2: 1: 7$ ). Figure. 4 show that the life of nodes steady increase along with the increased ratio of the $S_{1}$.

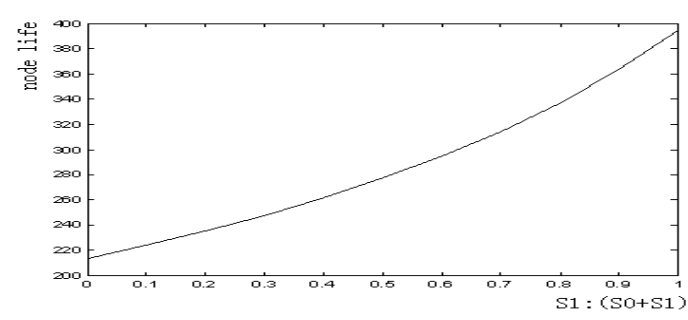

Figure.4 S1to node life influence

Figure.5 show that state ${ }^{S_{2}}$ has influence on working life of nodes, where, $T_{\text {work }}: T_{\text {idle }}=1: 5, S_{0}: S_{1}=1: 3$ and $S_{2}: S_{4}=2: 7$. The life of nodes sharply reduced along with the increased ratio of the state $S_{2}$. 


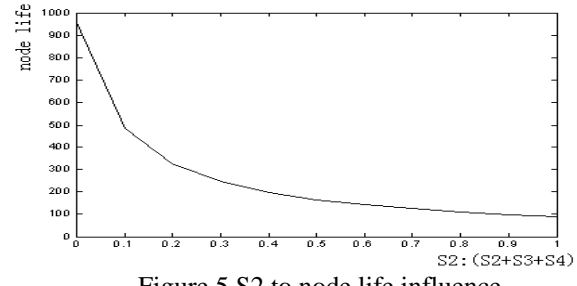

Figure.5 S2 to node life influence

Figure.6 show that the state $S_{3}$ has influence on working life of nodes, where, $T_{\text {work }}: T_{\text {idle }}=1: 5$, $S_{0}: S_{1}=1: 3, S_{2}: S_{4}=2: 7$. The life of nodes significantly prolonged along with the increased ratio of the state $S_{3}$.

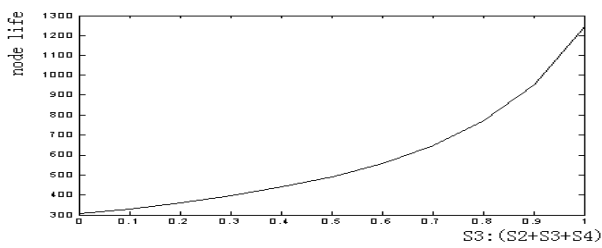

Figure.6 S3 to node life influence

Therefore, the state time of $S_{2}$ must be compressed to a minimum as far as possible in the nodes work, and when the data acquisition of sensors is over, the sensor module should be immediately shut down.

Effective network control strategy and routing algorithm is the key issue of the study in wireless sensor network measurement and control system, and the communication ability of nodes is an important basis to make effective control strategy and design routing algorithm. The energy consumption of wireless radio frequency module is much greater in the whole nodes, so the relationship between energy consumption and transmission distance is of great significance. The communication ability of nodes is researched by experiment.

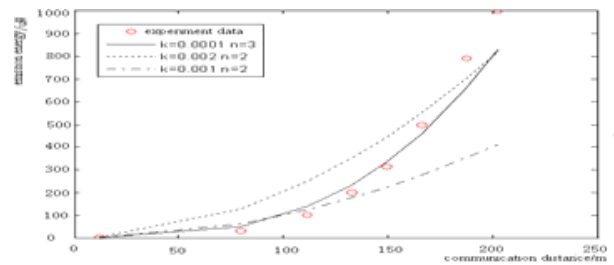

Figure.7 Node communication energy consumption

Due to the relationship of energy consumption and communication distance used in wireless sensor network at present is $P=k \cdot d^{n} \quad(2<\mathrm{n}<4)$. Considering the communication distance relationship in greenhouse application should not be too complex, Figure. 7 show the fitting effect of experiment data formula when $\mathrm{n}=2$ and $\mathrm{n}=$ 3. To fit effect, $P_{1}=0.0001 \cdot d^{3}$ when $\mathrm{n}=3$ was significantly better than $P_{2}=0.01 \cdot d^{2}$ and $P_{3}=0.02 \cdot d^{2}$ when $\mathrm{n}=2$. Therefore, the relation model of energy consumption and communication distance that is chosen is $P=k \cdot d^{3}$ in the paper.

The communication experiment is done with the last experiment (October 4, 2012) equipment on October 8 in 2012 in the same experimental conditions, and the four experiment data are processed and error analyzed. As is shown in Table.2, the mean value $\bar{\varepsilon}$ of communication distance, maximum error absolute value $\left|\xi_{\max }\right|$ and $\frac{\left|\xi_{\max }\right|}{\bar{\varepsilon}}$

maximum error percentage $\mathcal{E}$ were calculated in different emission power state. Table 2 calculation results show that the maximum error percentage $\frac{\left|\xi_{\max }\right|}{\bar{\varepsilon}}$ is smaller than $5 \%$, and meet the error requirements of the greenhouse WSN measurement and control system. The maximum error

$$
\underline{\mid \xi_{\max }} \mid
$$

of $\mathcal{E} \quad$ is $5.1 \%$, but this error appears when the emission power is $0.0036 \mathrm{~mW}$, and the maximum error absolute value $\left|\xi_{\max }\right|$ is only 0.63 meters, the error is allowed in greenhouse WSN measurement and control system that the resolution is $1 \mathrm{~m}$.

\section{TABLE.2 COMMUNICATION EXPERIMENT DATA ANALYSIS}

\begin{tabular}{|c|c|c|c|c|c|c|c|}
\hline \multirow[t]{2}{*}{ PA_LEVEL } & \multicolumn{3}{|c|}{2012.10 .4} & \multirow{2}{*}{2012.10 .8} & \multirow[t]{2}{*}{$\bar{\varepsilon}$} & \multirow{2}{*}{$\left|\varepsilon_{\mathbf{m a x}}\right|$} & \multirow{2}{*}{$\frac{\left|\xi_{\mathrm{max}}^{\mathrm{\varepsilon}}\right|}{\bar{\varepsilon}}$} \\
\hline & $9=00+$ & $13=000$ & $17=00+$ & & & & \\
\hline $0 \times 203$ & 12.0 & 12.5 & 11.5 & 12.5 & 12.13 & 0.63 & $5.1 \%$ \\
\hline $0 \times 07$ & 78.0 & 83.0 & 82.0 & 80.0 & 80.75 & 2.75 & $3.4 \%$ \\
\hline $\mathrm{o}_{\mathrm{z}} \mathrm{0 \textrm {B }}$ & $106.0 *$ & 110.0 & 110.5 & 111.5 & 109.5 & 3.5 & $3.2 \%$ \\
\hline OxOF & $131.0 \%$ & 128.0 & $132.0 \%$ & 127.5 & 129.63 & $2.37 \%$ & $1.8 \%$ \\
\hline $0 \times 13$ & 153.5 & 156.5 & $146.0 \%$ & 148.5 & 151.13 & 5.37 .7 & $3.6 \%$ \\
\hline $0 \times 17$ & $170.0 \%$ & 173.50 & 172.50 & 166.0 & 170.5 & 4.5 & $2.6 \%$ \\
\hline $0 \times 19.0$ & 185.00 & 192.0 & 187.50 & 191.5 & 189.00 & 4.0 & $2.1 \%$ \\
\hline $0 \times 1 F$ & 201.5 & 206 & 200.0 & 210.5 & 204.5 & 6.0 & $2.9 \%$ \\
\hline
\end{tabular}

The experiment data in Table.2 is fitted by polynomial, and results are shown in Figure.8.

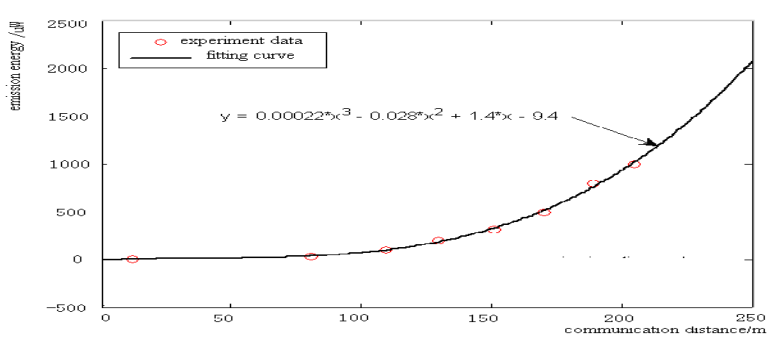

Figure.8 Cubic polynomial fitting 
On the basis of cubic polynomial fitting formula $P_{0}=0.00022 \cdot d^{3}-0.028 \cdot d^{2}+1.4 \cdot d-9.4$ when $k$ is different value, the fitting curve $P=k \cdot d^{3}$ is fitted. When $k$ is different coefficient, the error of fitting curve is analyzed in Table 3 , and the results are as follows.

1.When $\mathrm{k}=0.00008$ and PA_LEVEL is out of $0 \mathrm{X} 1 \mathrm{~F}$, the maximum error absolute value $\left|\xi_{\max }\right|$ appeared, The $\left|\xi_{\text {max }}\right|$ value of the rest four curve are all appear when PA_LEVEL value is 0X03, and when PA_LEVEL value is $0 \times 03$ and $\mathrm{k}=0.00008$, the error of curve is maximum . Therefore, the fitting model of energy consumption and communication distance is suitable for application scenario that the communication distance is longer than 50 meters.

2. The $\left|\sum_{i=1}^{8} \xi_{i}\right|$

then decreases when $\mathrm{k}$ is varied error value is bigger when $\mathrm{K}=0.00008$. When $\mathrm{k}=0.0001$ and $\mathrm{k}=0.00011$, the $\left|\sum_{i=1}^{8} \xi_{i}\right|$ value is smaller than the remaining three, and the minimum value appear when $\mathrm{k}=$ 0.0001 . Therefore, the coefficient $\mathrm{k}$ value range of $P=k \cdot d^{3}$ is $0.00009<k<0.00012$.

3. According to the distribution of positive and negative of the error $\xi_{i}$, when $\mathrm{k}=0.0001$, the value of $\xi_{i}$ is four positive and four negative, and it is even distributed in both sides of fitting curve, the distribution is more ideal when $\mathrm{k}=$ 0.00011 .

TABLE.3 DIFFERENT FITTING CURVE ERROR ANALYSIS

\begin{tabular}{|c|c|c|c|c|c|c|}
\hline PA_LEVEI & $a_{\alpha}$ & 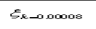 & 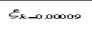 & $E_{s-a .6001}$ & 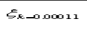 & 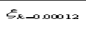 \\
\hline $0 \times 03$ & 12.13 & -23.4389 & -22.0605 & -20.8803 & -10.3567 & -18.9423 \\
\hline $0: 807$ & 80.75 & 7.0694 & 0.9061 & 12.3510 & 14.4898 & 16.3840 \\
\hline $0=0 \mathrm{~B}$ & 109.5 & 1.9783 & 0.1256 & 0.7000 & 12.8271 & 15.5964 \\
\hline $0=0 F$ & 129.63 & -6.0909 & -0.8656 & 3.6379 & 7.5778 & 11.0669 \\
\hline $05 \times 13$ & 151.13 & -6.7790 & -0.6994 & 4.5403 & 9.1242 & 13.1838 \\
\hline $0 \times 17$ & 170.5 & -13.7016 & -6.6098 & -0.4976 & 4.8496 & 9.5851 \\
\hline 02819 & 189.0 & -25.5420 & -17.2821 & -10.1632 & -3.9352 & 1.5803 \\
\hline $0 \times 1 F$ & 204.5 & -27.5794 & -18.6443 & -10.9435 & 4.2064 & 1.7599 \\
\hline \multicolumn{2}{|c|}{$\begin{array}{l}\text { Maximann errax } \\
\text { absolute value }\left|s_{\max }\right|\end{array}$} & 27.5794 & 22.0695 & 20.2893 & 19.3567 & 18.9423 \\
\hline \multicolumn{2}{|c|}{$\left.\right|_{i=1} ^{n}=1$} & 94.085 & 50.139 & 12.2644 & 20.3702 & 50.2141 \\
\hline
\end{tabular}

According to the above analysis, and this paper take $P_{1}=0.0001 \cdot d^{3}$ as communication energy formula. The fitting curve is shown in Figure.9.

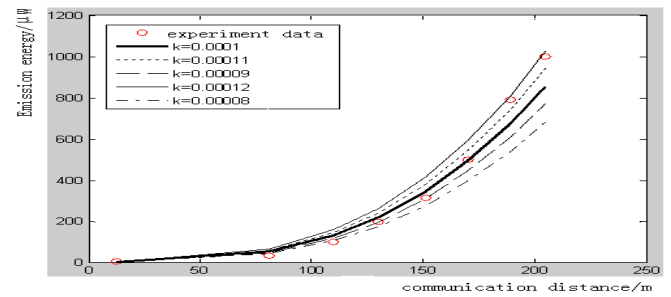

Figure.9 Three fitting curve of different coefficient

\section{CONCLUSION}

In this paper, the energy management strategy of nodes in wireless sensor network was specifically analyzed, and the energy consumption of low power consumption sensor nodes in different working status, the switch time and energy consumption of the working state, as well as the sensor nodes communication ability were all studied by experiment. The experiment and simulation results show that the energy consumption and three cubed of communication distance for nodes is direct proportion relationship, and the moving network structure work mode of the convergent nodes can greatly shorten the communication distance of sensor nodes and save energy consumption.

\section{ACKNOWLEDGEMENTS}

This work was financially supported by HuaiHai Institute of Technology Natural Science Foundation (Z2011004),

\section{REFERENCES}

[1] WANG X, MA J M, WANG,S,et cl. Dynamic energy management with improved panicle filter prediction in wireless sensor networks[A] . International Conference on Intelligent Computation[C]. 2007. 251-262.

[2] SINHA A, CHANDRAKASANA. Dynamic power management in wireless sensor networks[J]. IEEE Design \& Test of Computers. 2001, 18(2) : 62-74.

[3] ENINI L, BOGLIOLO A, MICHELI G . A survey of design techniques for system-level dynamic power management[J]. IEEE Transactions On Very Large Scale Integration(VLSI)Systems, 2000. 8(3) : 299-316.

[4] GHARAVI H, KUMAR S P. Special issues on sensor networks and applications[J]. Proceedings of the IEEE, 2003, 91(8) : 11511153.

[5] KUBISCH M, KARL H, WOLISZ A, et al. Distributed algorithm for transmission Power control in wireless sensor networks[C]. Proc of IEEE Wireless Communications and Networking Conference. $2003:$ 16-20.

[6] Chuan Lin, Yan-Xiang He, Naixue Xiong.An Energy-Efficient Dynamic Power Management in Wireless Sensor Networks[J]. ISPDC-06,IEEE, 2006, Pages:148-154.

[7] Guo.Dong. Gu: Research on Energy Management of Wireless Sensor Network of Monitoring System in Greenhouse[D]. Jiangsu university, 2008 